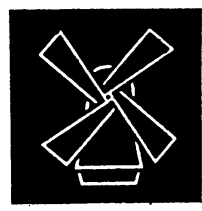

\title{
PROBLEMI dell'informazione
}

Anno I, n. 3, luglio-settembre 1976

PAOLO MURIALDI, La paura dell'informazione

\section{La RAI-TV nella riforma}

PAOLO BARILE, Un monopolio riformato a metà. Un primo rendiconto sull'applicazione della legge di riforma

GIOVANNI BECHELLONI, La giostra delle notizie. A proposito della nuova informazione della RAI-TV

MARINA TARTARA, Ricostruire un'azienda

GIANCARLO CARCANO, La Sipra: da un'isola all'arcipelago pubblicitario

GRAZIA PRIULLA CAZZOLA - SALVATORE SCIBILIA, La prima settimana di informazione riformata. Confronto fra TG1 e TG2

La stampa senza riforma

VALERIO CASTRONOVO, La stampa senza riforma. II nuovo pericolo

GIORGIO GALLI, Stampa e cultura politica

\section{Saggi e ricerche}

\section{Rassegne}

\section{Notiziario}

Escono quattro fascicoli ogni anno. Un fascicolo L. 2.500. Abbonamento annuo: L. 9.000 per I'Italia, L. 10.000 per l'estero.

\section{LO SPETTATORE INTERNAZIONALE}

Anno XI, number 1, January-March 1976

Investiment and Labour in the Mediterranean: The IAI Project, edited by Roberto Aliboni

JOSEPH SASSOON, Labour and Capital Movements in the Mediterranean area

GIACOMO LUCIANI, The Multinational Corporation's Strategy in the Mediterranean

Special Feature. Annual Report for 1975 and Program for 1976

Escono quattro fascicoli ogni anno. Abbonamento annuo: L. 5.000 per I'Italia, L. 6.000 per l'Europa, L. 7.000 per gli altri Paesi (air-mail). 


\section{Novità maggio-agosto nelle edizioni del Mulino}

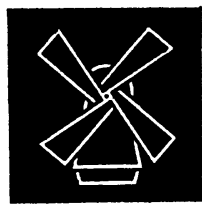

Nella collana " Universale Paperbacks il Mulino "

JEAN VIAL, Lo sviluppo della società industriale dal 1815 ad oggi pp. 304 , L. 2.800

MASSIMO TEODORI, Storia delle nuove sinistre in Europa pp. 680 , L. 4.800

JEAN BAUDRILLARD, La società dei consumi pp. 296, L. 2.800

CARLO FELICE CASULA, Cattolici comunisti e sinistra cristiana pp. 340 , L. 3.000

MORTON DEUTSCH-ROBERT M. KRAUSS, La psicologia sociale contemporanea

pp. 320 , L. 3.500

Nella collana "Saggi "

GUGLIELMO FORNI, Alienazione e storia. Saggio su Rousseau pp. 160 , L. 3.000

GEORGES CANGUILHEM, La conoscenza della vita pp. 270, L. 6.000

RAINER EISFELD, II pluralismo fra liberalismo e socialismo pp. 280 , L. 5.000

HARALD WEINRICH, Metafora e menzogna: la serenità dell'arte pp. 248 , L. 6.000

Nella "Collezione di testi e di studi"

TEUN A. VAN DIJK, Per una poetica generativa pp. 328, L. 6.000

JURGEN RUESCH-GREGORY BATESON, La matrice sociale della psichiatria

pp. 360 , L. 7.500 


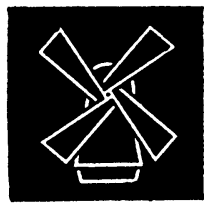

\section{Rassegna Italiana}

\section{di Sociologia}

Anno XVII, n. 2, aprile-giugno 1976

PERCY ALLUM, II caso italiano

\section{Saggi e ricerche}

NICOLO ADDARIO, Sulla classe dominante nelle società socialiste CARLO TRIGLIA, Sviluppo, sottosviluppo e classi sociali in Italia

\section{Materiali di ricerca}

FRANCO CAZZOLA, I pilastri del regime. Gli enti pubblici di sicurezza sociale

\section{Note critiche}

MILLY BUONANNO, Antropologia e condizione della donna

ALESSANDRO CAVALLI, Perché non si parla né bene né male dell'inferma scienza

CARLO G. ROSSETTI, A proposito de * L'oggetto della teoria generale di T. Parsons *

Escono 4 fascicoli ogni anno. Un fascicolo L. 2.500. Abbonamento annuo: L. 9.000 per I'Italia, L. 10.000 per l'estero.

\section{RIVISTA DI ECONOMIA E POLITICA INDUSTRIALE}

Anno II, n. 1, gennaio-aprile 1976

FRANCESCO CESARINI, II mercato del reddito fisso in Italia di fronte alla nuova crisi

FABIO GOBBO, Una riconsiderazione dei problemi della crescita e delle dimensioni dell'impresa

PIERLUIGI ALZONA, Redditività e inflazione

GIORGIO DONNA, Nuove strategie per l'industria dell'auto

GIULIANO AMATO, Le partecipazioni statali: cenni descrittivi del sistema e della sua evoluzione

NINO ANDREATTA, Le partecipazioni statali domani

Escono 3 fascicoli all'anno. Un fascicolo L. 3.000. Abbonamento annuo: L. 8.000 per I'Italia, L. 9.000 per l'estero. 


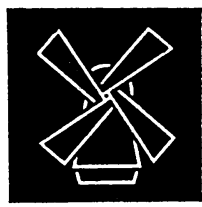

\section{POLITICA \\ DEL DIRITTO}

Anno VII, n. 3/4, agosto 1976

\section{Editoriale}

Commenti

Saggi

MARC GALANTER, Perché gli abbienti si avvantaggiano. Riflessioni sui limiti del riformismo giuridico

SANDRO AMOROSINI, I servizi di sicurezza

ENRICO PATTARO, Sui mezzi di controllo sociale: diritto, morale e politica

GIORGIO GHEZZI, L'intervento degli enti locali nella politica culturale

Documenti e discussioni

\section{Notizie}

Escono sei fascicoli ogni anno. Un fascicolo L. 2.000. Abbonamento annuo: L. 10.000 per l'Italia, L. 12.000 per l'estero.

\section{LA QUESTIONE CRIMINALE}

Anno II, n. 1, gennaio-aprile 1976

Studi

FRANCO BRICOLA, Partecipazione e giustizia penale (Le azioni a tutela dell'interesse collettivo)

J.C. WEINBERGER, P. JAKUBOWICZ, PH. ROBERT, II declino del diritto... come strumento di controllo sociale

\section{Dibattiti}

GAETANO INSOLERA, LUIGI STORTONI, Un'altra legge " speciale ": la legge sulla droga

\section{Documenti}

NICOLA MAZZACUVA, La questione penale nella " critica sociale * (1898-1970); materiali e prime indicazioni per una ricerca

\section{Ricerche}

DARIO MELOSSI, Ricerca in tema di mass-media e politica criminale

Escono tre fascicoli all'anno. Un fascicolo L. 3.000. Abbonamento annuo: L. 8.000 per l'Italia, L. 9.000 per l'estero. 


\section{Le riviste edite dal Mulino}

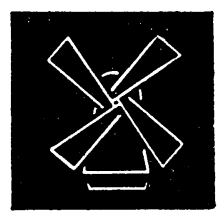

\section{IL MULINO}

Anno XXV, n. 3, maggio-giugno 1976

\section{Saggi}

PIETRO SCOPPOLA, Dopo il 20 giugno

ARTURO PARISI-GIANFRANCO PASQUINO, 20 giugno: struttura politica e comportamento elettorale

GIUSEPPE DE RITA, Verso una parziale riprivatizzazione dei consumi sociali?

UMBERTO ROMAGNOLI, Costituzione economica e cooperative di lavoro

SIMON SERFATY, Gli Stati Uniti e il tripartitismo in Francia e in Italia, 1945-1947

\section{Dibattito}

ANTONIO GHIRELLI, Comunismo e società: la classe dirigente del $\mathrm{PCl}$ vent'anni dopo

Escono 6 fascicoli all'anno. Un fascicolo: L. 2.000. Abbonamento annuo: L. 10.000 per l'Italia, L. 12.000 per l'estero.

\section{Storia}

\section{contemporanea}

Anno VII, n. 2, aprile-giugno 1976

\section{Saggi}

BRUNO TOBIA, La diffusione in Italia del movimento "Clarté " di Henri Barbusse

ROSELLINA MARIANI, I convegni di Weimar

\section{Note e discussioni}

LEONARDO RAPONE, Movimenti fascisti e classi sociali nell'analisi di Lev D. Trotskji

CLAUDIO NATOLI, L'internazionale comunista, il fronte unico e la lotta contro il fascismo in Italia e in Germania (1919-1923)

\section{Rassegne}

ALDO ALBONICO, Portogallo: un accostamento storico

Escono quattro fascicoli ogni anno. Un fascicolo normale L. 3.000 . Abbonamento annuo: L. 10.000 per l'Italia, L. 12.000 per l'estero. 


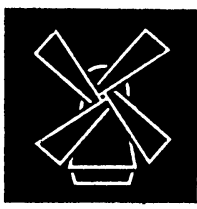

LINGUA

E STILE

Anno XI, n. 2, aprile-giugno 1976

\section{Saggi}

FRANCESCO ANTINUCCI, Le due anime di Chomsky

HANS-HEINRICH BAUMANN, Ueber die dreifache Wurzel der Idee zu einer Implikativen Typologie

GIUSEPPE A. SANSONE, Equilibri ritmici garcilasiani: il sonetto XVII ANTONIO PORCU, La "Vita " dell'Alfieri come vicenda linguistica Problemi

Escono 4 fascicoli ogni anno. Un fascicolo L. 2.500. Abbonamento annuo: L. 9.000 per l'Italia, L. 10.000 per l'estero.

\section{GIORNALE ITALIANO DI PSICOLOGIA ITALIAN JOURNAL OF PSYCHOLOGY}

Anno III, n. 2, agosto 1976

\section{Discussioni}

HENRI TAJFEL, La psicologia sociale e $i$ processi sociali

DARIO ROMANO, CLAUDIO CONTI, Verso uno psicologia sociale " concreta $*$ ?

\section{Rassegne}

LUCIA LUMBELLI, Problemi di confine tra psicologia e pedagogia Contributi

PAOLO RENZI, ALBERTO OLIVERIO, A genetic analysis of discriminated Bar-press avoidance in the mouse

DARIO SALMASO, FRANCO DENES, GIORGIO DE STAVOLA, Intermispheric differences in attention to novelty

WILLEM DOISE, HANNS-DIETRICH DANN, New theoretical perspectives in the experimental study of intergroup relations

CARLO UMILTA, FRANCESCA SIMION, RAY HYMANN, The repetition effect for verbal and non-verbal stimuli in the two visula field

GAETANO KANIZSA, GIORGIO TAMPIERI, Environmental and retinal frame of reference in visual perception

Escono tre fascicoli all'anno. Un fascicolo L. 3.000. L'abbonamento annuo: L. 8.000 per l'Italia, L. 9.000 per l'estero. 
Responsabile: Giovanni Sartori - Stampa: Grafiche BG - Rastignano, Bologna. Registrato al n. 4110 presso la Cancelleria del Tribunale Civile Penale di Bologna, il 14-12-1970 


\section{II supplemento}
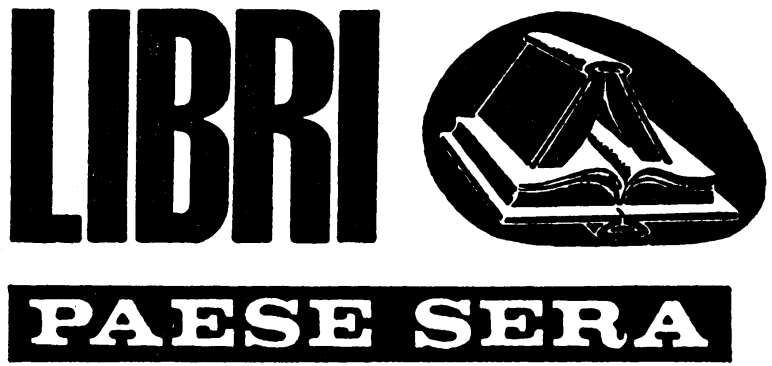

è l'unica rassegna settimanale dedicata ad una organica informazione sulle novità librarie italiane e straniere.

Insieme ad un elenco delel opere pubblicate dai principali editori troverete, in ogni numero, articoli, recensioni e note di collaboratori italiani e stranieri dedicate ai libri di maggiore interesse in ogni campo:

Arte, letteratura, scienze sociali e scienze umane, filosofia, scienza, storia, attualità, politica, teatro e libri per ragazzi.

Esce ogni venerdi.

Potete abbonarvi direttamente al supplemento LIBRI PAESE SERA utilizzando il c/c n. 1-30642 e indirizzando la richiesta all'Ufficio Abbonamenti in Via dei Taurini 19-00185 ROMA.

Abbonamento: sei mesi, L. 2.200; un anno, L. 4.000 


\section{CRONACA DELTE COSE UTHI}

\section{Informazioni su cose, attività, servizi? Su Pagine Gialle. Pagine Gialle: una cronaca che dura un anno intero. E risponde - per 365 giorni - alle domande di tutti i giorni.}

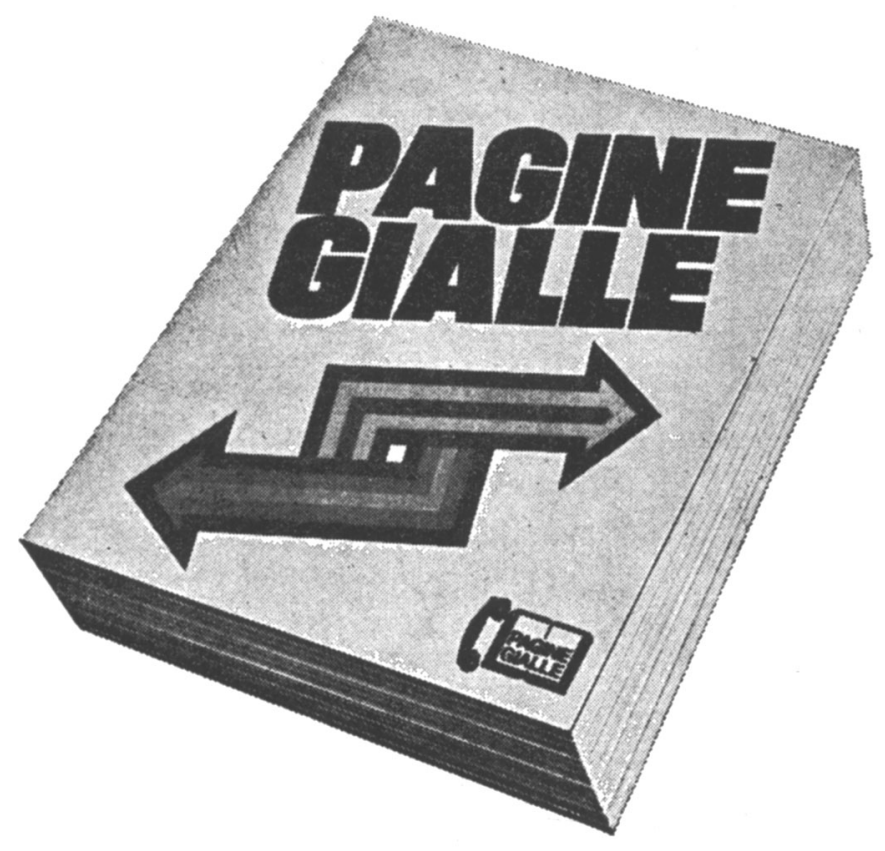


Novità nella collana

"Universale Paperbacks il Mulino"

Jean Baudrillard

La società dei consumi

L. 2.800

Massimo Teodori

Storia

delle nuove sinistre

in Europa (1956-1976)

L. 4.800

Carlo Felice Casula

Cattolici-comunisti

e sinistra cristiana (1938-1945)

L. 3.000

IL MULINO

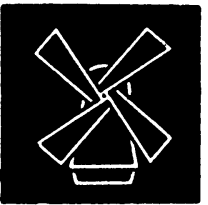




\title{
La stampa quotidiana tra crisi e riforma
}

\author{
a cura di \\ Paolo Barile e Enzo Cheli
}

1. Vanna Console, I lavori preparatori dell'Assemblea costituente in tema di stampa periodica

2. Ugo De Siervo, Recenti linee di tendenza degli interventi legislativi e governativi in materia di stampa

3. Paolo Caretti, Le agenzie di informazione in Italia

4. Emily Rawlence, Le agenzie di informazione estere

5. Stefano Grassi, Gli uffici stampa degli organi pubblici

6. Enzo Grassi, II prezzo amministrato della stampa quotidiana in Italia

7. Franco Bassanini, Le provvidenze all'editoria e il pluralismo dell'informazione problemi di attuazione della legge 6 giugno 1975, n. 172

8. A. Orsi Battaglini, Ente nazionale cellulosa e carta

9. Roberto Zaccaria, La pubblicità sulla stampa: ipotesi per una disciplina legislativa

10. Carlo Macchitella e Domenico Sorace, I problemi della distribuzione

11. S. Merlini, Ordine dei giornalisti, organizzazione sindacale e "professionalità " del lavoro giornalistico: profili di costituzionalità

12. Pietro Zanelli, Aspetti particolari del trattamento economiconormativo dei giornalisti

13. Marcello Pedrazzoli, Appunti sulla "tendenza " dell'imprenditore con particolare riguardo al lavoro giornalistico

14. M. Cristina Grisolia, I comitati di redazione e i problemi della partecipazione dei giornalisti alla gestione dell'impresa

15. Piergaetano Marchetti, Problemi in tema di organizzazione giuridica dell'impresa giornalistica

16. Giovanni Spinoso, Le esperienze dei nuovi giornali locali in Italia

17. Susanna della Felice e Paolo Golini, II Press Council e analoghe esperienze

I L M ULINO

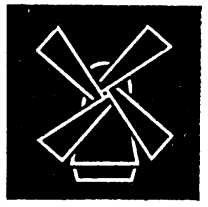


Nella "Nuova Collana Storica"

Cristopher Hill

Le origini intellettuali

della rivoluzione

inglese

Jeffry Kaplow

I lavoratori poveri

nella Parigi

prerivoluzionaria

I L M U LINO

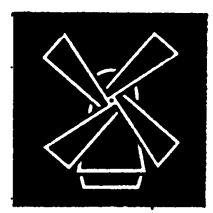




\section{Giorgio Fuà \\ Occupazione \\ e capacità produttive: la realtà italiana}

Universale Paperbacks il Mulino

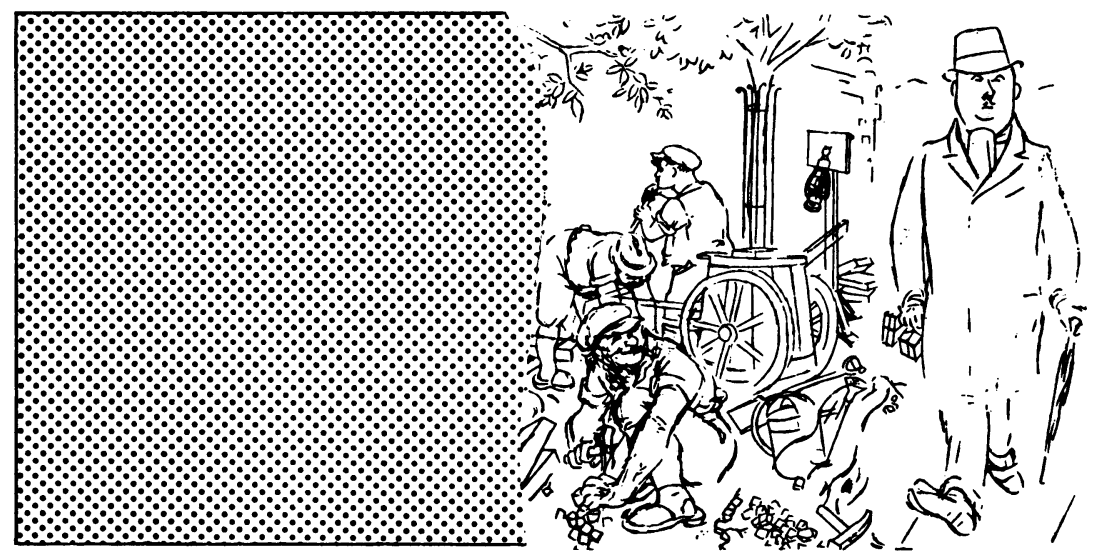

Un volume di 125 pagine, L. 1.500 


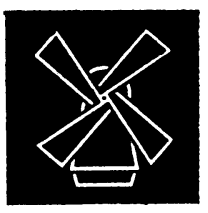

Nella "Nuova collana storica "

CHRISTOPHER HILL, Le origini intellettuali della Rivoluzione inglese pp. 456 , L. 10.000

Nella collana "La nuova scienza"

MARIO NIGRO, Giustizia amministrativa

pp. 320 , L. 5.000

DENNIS SWANN, L'economia del Mercato comune pp. 280 , L. 4.000

FRANK RESTLE, I modelli matematici in psicologia pp. 164 , L. 3.000

\section{Nella collana "Problemi e prospettive"}

La tipologia linguistica, a cura di PAOLO RAMAT

pp. 352 , L. 6.000

Magia e scienza nella civiltà umanistica, a cura di CESARE VASOLI pp. 304, L. 6.000

Evoluzione: biologia e scienze umane, a cura di GIULIANO PANCALDI

pp. 320 , L. 6.000

La dialettica nel pensiero contemporaneo, a cura di VALERIO VERRA pp. 362 , L. 6.000

La psicolinquistica, a cura di FRANCESCO ANTINUCCI e CRISTIANO CASTELFRANCHI

pp. 428 , L. 8.000

Agiografia altomedievale, a cura di SOFIA BOESCH GAJANO pp. 312 , L. 6.000

Nella collana "Temi e discussioni"

La stampa quotidiana tra crisi e riforme, a cura di PAOLO BARILE e ENZO CHELI

pp. 722 , L. 10.000 
Nella collana "Problemi e prospettive: storia ", una nuova serie dedicata a:

\section{Istituzioni e società nella storia d'Italia}

E' uscito il volume quinto:

Gli apparati statali

dall'Unità al fascismo

a cura di

ISABELLA ZANNI ROSIELLO

Sono in preparazione:

I. Ordinamenti feudali e signorili e Comuni a cura di

GABRIELLA ROSSETTI

II. Dalla crisi del Comune allo Stato del Rinascimento a cura di

GIORGIO CHITTOLINI

III. Gli Stati regionali

a cura di

ELENA FASANO GUARINI

IV. Gli Stati pre-unitari

a cura di

NICOLA RAPONI

I L M ULINO

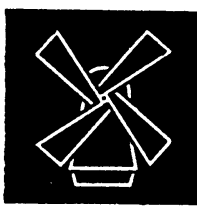




\section{NEI PROSSIMI NUMERI}

B. Bartolini, L'influenza delle subculture sul voto democristiano $e$ comunista

M. Caciagli, Il dibattito metodologico dei politologi tedeschi

R.A. Dahl, Il pluralismo rivisitato

P. Flora et al., La politica della sicurezza sociale in Europa

L. Morlino, Un manuale di scienza politica

G. Pasquino, Militari e politica: rassegna di studi

G. Sani, Protesta, insoddisfazione e mutamento politico in Italia

S. Passigli, La mappa del potere locale 
